\title{
Cómo hacer ciencia con aparatos. Un enfoque materialista de la fisica cuántica ${ }^{1}$
}

\author{
CARLOS MADRID \\ Universidad Complutense de Madrid \\ carlos.madrid@educa.madrid.org
}

Recibido: 07.04.2009

Aceptado: 02.11.2009

\begin{abstract}
Restrinjamos nuestra atención a las operaciones de laboratorio que suministran al científico indicios parciales pero concretos de lo que ya ha visto. [...] Tras una revolución científica, muchas antiguas medidas y manipulaciones se hacen irrelevantes y son reemplazadas por otras. Uno no aplica las mismas pruebas al oxígeno que al aire deflogistizado. Sin embargo, los cambios de este tipo no son nunca totales. [...] Gran parte del lenguaje del científico y la mayoría de sus instrumentos de laboratorio siguen siendo los mismos que antes. Como consecuencia, la ciencia posrevolucionaria incluye inevitablemente muchas de las mismas manipulaciones, realizadas con los mismos instrumentos y descritas en los mismos términos que empleaba su predecesora prerrevolucionaria.
\end{abstract}

T. S. Kuhn, La estructura de las revoluciones científicas, pp. 129-130.

\section{LOS TRES COMPONENTES DEL GIRO «PRAXIOLÓGICO»}

El nuevo giro que ha tomado el estudio de la ciencia, interesándose por el experimento y la práctica científica, es producto de la composición de tres líneas de investigación: la sociológica, la histórica y, finalmente, la filosófica. Dentro de la línea sociológica nos encontramos con la Sociología del Conocimiento Científico. Y, por su parte, dentro de la línea histórico-filosófica, con el Nuevo Experimentalismo.

La Sociología del Conocimiento Científico (SCC) nace, durante la década de 1970, dentro de la Escuela de Edimburgo-Bath. Los nombres de los padres son bien conocidos: Barry Barnes, David Bloor, Harry Collins... A sus trabajos seminales se uniría el estudio etnográfico Laboratory Life de Bruno Latour y Ste-

1 Quiero agradecer a los dos evaluadores anónimos su valiosa lectura «teoreticista» y «experimentalista», respectivamente, del artículo.

EMPIRIA. Revista de Metodología de Ciencias Sociales. N. ${ }^{o}$ 18, julio-diciembre, 2009, pp. 147-170. ISSN: $1139-5737$ 
ve Woolgar, publicado en 1979, así como el estudio de laboratorio The Manufacture of Knowledge de Karin Knorr Cetina, publicado en 1981. La SCC analiza la ciencia como proceso social, atendiendo a cómo los científicos construyen el conocimiento científico. Desde los años 80, la SCC ha conocido un paulatino pero lento desarrollo, debido — según asegura Andrew Pickering (1992: 6), uno de sus principales representantes - a la ausencia de unos buenos estudios de campo que terminen por alejar la imagen excesivamente abstracta de la ciencia de algunos sociólogos del conocimiento.

Al tiempo que la SCC comenzaba a llamar la atención sobre los aspectos prácticos de la ciencia, una nueva corriente surgía en las aguas de la filosofía de la ciencia: el Nuevo Experimentalismo (NE). La filosofía analítica de la ciencia ha venido dedicando poca atención a la práctica científica. De hecho, incluso el giro «historicista» mantiene, al igual que la Concepción Heredada o Sintáctica y la actual Concepción Semántica, una fuerte orientación teorética. Por su tendencia a centrarse en la historia de las teorías científicas, Kuhn constituye - como Carnap o Popper - uno de los máximos exponentes de la miseria del «teoreticismo», por decirlo con Ferreirós y Ordóñez (2002). Sin embargo, a comienzos de los años 80, algo estaba a punto de cambiar. Representing and Intervening de Ian Hacking (1983) es el hito que marca el cambio de rumbo en la filosofía de la ciencia. Hacking enfatizó que la ciencia es hacer (intervenir) y no sólo conocer (representar), reivindicando el papel del experimento en ciencia. La concepción de la ciencia que acuñó Hacking fue bautizada con el nombre de new experimentalism por Robert Ackermann (1989). Dentro del NE, que proclama una suerte de retorno a Francis Bacon, se cuentan los trabajos pioneros de Nancy Cartwright (1983) o Allan Franklin (1989), que han contribuido a invertir la tradicional jerarquía de la teoría sobre el experimento.

Estas ideas que estaban abriéndose paso en los ámbitos sociológico y filosófico fueron, precisamente, el caldo de cultivo de una nueva historiografía de la ciencia. Si bien, la historia de la ciencia entró en el tema algo antes que la filosofía, y casi en simultáneo con la sociología, con precedentes como la obra de John L. Heilbron (1979) ${ }^{2}$. No tardando mucho, autores como Steven Shapin y Simon Schaffer (1985), Allan Franklin (1986) o Peter Galison (1987) comenzaron a escribir la historia de la experimentación e instrumentación en física, química, biología, etc. Estos estudios nos han mostrado al experimento decidiendo entre dos teorías competidoras, pidiendo una nueva teoría, confirmando o refutando otra, sugiriendo formas matemáticas y hasta descubriendo nuevos componentes de la materia. Nos han enseñado que la práctica y el experimento tienen vida propia, independiente de la teoría.

El propósito de este artículo es analizar las relaciones metodológicas de esta nueva historia de la ciencia con la sociología y la filosofía de la ciencia. En primer

${ }^{2}$ Heilbron (1989 y 1999) ha mantenido posteriormente este toque, al continuar poniendo el énfasis principal en los aparatos (las catedrales como observatorios solares) y su invención (el diseño y la construcción del laboratorio de Lawrence en Berkeley).

EMPIRIA. Revista de Metodología de Ciencias Sociales. N. ${ }^{\circ}$ 18, julio-diciembre, 2009, pp. 147-170. ISSN: 1139-5737 
lugar, vamos a explicar los presupuestos gnoseológicos de la historia experimentalista de la ciencia, que están ligados a las tesis de la SCC y del NE. Estamos en condiciones de adelantar a grandes rasgos las premisas gnoseológicas de la nueva historia de la ciencia: a) predominio del idioma performativo sobre el idioma representacional en ciencia; b) el científico es un sujeto técnico-operatorio; c) la ciencia comprende una cultura material; y d) los aparatos e instrumentos de laboratorio son los contextos determinantes de las ciencias. En especial, a lo largo de esta primera parte del artículo, vamos a contrastar continuamente tres concepciones de la ciencia confluyentes pero de orígenes muy distintos: la sociológica de Andrew Pickering, la epistemológica de Ian Hacking y la ontológica de Gustavo Bueno. Ya veremos por qué. En segundo lugar, recíprocamente, vamos a abordar las implicaciones históricas del NE y la SCC, esto es, cómo pueden la filosofía y la sociología servir de heurística a la historia. El estudio del papel que los aparatos juegan en física cuántica ilustrará nuestra tesis principal: la historia de la ciencia es, en gran medida, la historia de sus aparatos. Habida cuenta todo ello de que - parafraseando el célebre lema lakatosiano- la filosofía y la sociología sin historia de la ciencia están vacías, pero la historia de la ciencia sin filosofía y sociología está ciega.

\section{DE LA ACADEMIA AL LABORATORIO, PASANDO POR EL TALLER}

Con motivo del 80 cumpleaños de Sir Karl Popper, Ian Hacking quiso tratar de arrumbar la tradicional hegemonía de la teoría sobre la práctica en ciencia, porque, precisamente, la filosofía popperiana representaba un ejemplo estrella de la filosofía de la ciencia dominada por la teoría contra la que Hacking despotrica. Para Popper, si tras una catástrofe universal la Humanidad conservaba intactas las bibliotecas, aunque perdiera todas las máquinas, los hombres se sobrepondrían, porque averiguarían cómo fabricarlas una a una leyendo libros. Sin embargo, para Hacking, si la Humanidad estuviera condenada a una cultura meramente libresca, sin ningún conocimiento práctico sobre maquinaria, sobrevendría una Edad Oscura, puesto que los hombres lo pasarían muy mal para hacer que algo funcionara. Hacking dio la vuelta a la historia: si tras una catástrofe universal la Humanidad conserva las máquinas, aunque carezca de libros y manuales, los hombres tendrán mucho más fácil salir adelante. Teniendo máquinas en buen estado, las personas con el tipo de habilidad que hoy tienen los obreros y artesanos acabarían por averiguar qué hacen las máquinas y cómo lo hacen. No en vano, Ortega y Gasset escribió, en Meditación de la técnica, que el hombre era el animal maquinista por excelencia. «La lección es — concluye Hacking (1996: 303)—: hay que pensar en la práctica, no en la teoría».

La filosofía «teoreticista» de la ciencia anima a decir, con Popper, que todo nuestro saber se reduce a un mero conjeturar; pero ni mucho menos. Basta argumentar ad hominem: la explosión de una bomba atómica hace saltar por los

EMPIRIA. Revista de Metodología de Ciencias Sociales. N. ${ }^{\circ}$ 18, julio-diciembre, 2009, pp. 147-170. ISSN: 1139-5737 
aires cualquier clase de escepticismo (nunca mejor dicho). Aún así, la mayoría de las discusiones post-kuhnianas siguen ancladas en una concepción de la ciencia casi exclusivamente logoteórica, que ignora en gran medida su carácter técnico y operativo. Incluso la vigente concepción de la ciencia de Bas Van Fraassen (1996) está completamente dominada por la teoría, quedando la práctica y el experimento en un discreto segundo plano, condenados a únicamente decidir si una teoría ya articulada es o no es adecuada empíricamente. Sin embargo, no se produce ciencia intercambiando teorías sino actuando sobre la realidad. Para conocer científicamente hay que transformar el mundo en una probeta o en un ciclotrón. Lo que los científicos manejan o manipulan, equipados con las teorías y los modelos, no son otras teorías u otros modelos, sino la realidad misma por medio de los aparatos. La ciencia comprende un saber hacer, sin el cual no hay observación, medición, experimentación o cálculo. Los científicos no sólo formulan teorías o hipótesis de que deducir predicciones, dedican la mayor parte del tiempo a experimentar, construir y revisar modelos, manejar aparatos, diseñar artefactos y accionar máquinas. Los científicos son, principalmente, agentes que hacen cosas con cosas (incluso plantear leyes consiste en una práctica escrita). A la manera que la arquitectura consiste - por así decir - en mover grandes piedras empleando la geometría y las grúas, la ciencia consiste en manejar o manipular diversas entidades reales empleando la teoría y los aparatos.

En suma, la ciencia no es sólo un cuerpo de conocimiento, de proposiciones que buscan representar el mundo; porque el científico no sólo compone teorías sino que también cambia e interviene la realidad con los instrumentos y artilugios que construye. En The Mangle of Practice, el sociólogo Andrew Pickering (1995: 5-6) introduce la distinción entre el idioma representacional y el idioma performativo $^{3}$ a la hora de pensar la ciencia:

El idioma representacional concibe la ciencia, sobre todo, como una actividad que busca representar la naturaleza, producir conocimiento que mapeé, refleje o se corresponda con cómo el mundo es realmente. [...] Sin embargo, dentro de una concepción expandida de la cultura científica — una que vaya más allá de la ciencia como conocimiento para incluir la dimensión material, social y temporal de la ciencia- se hace posible imaginar que la ciencia no es sólo representación. Uno puede comenzar con la idea de que el mundo no está compuesto, en primera instancia, de hechos y observaciones, sino de «agencia». El mundo, quiero decir, está continuamente haciendo cosas.

La filosofía de la ciencia tradicional ha sido incapaz de superar la barrera del idioma representacional; y, por su parte, la SCC ha sido, a lo sumo, semi-performativa (Pickering 1995: 10). Sin embargo, Pickering subraya que es necesario cambiar de marco para entender cabalmente la práctica científica: el idioma performativo predomina en la ciencia. Este idioma nos aleja de concebir la

3 Traduzco el término «idiom» que utiliza Pickering en su sentido figurado de estilo, lenguaje o idioma, prefiriendo este último por cuanto entronca mucho mejor con la idea de uso social.

EMPIRIA. Revista de Metodología de Ciencias Sociales. N. ${ }^{\circ}$ 18, julio-diciembre, 2009, pp. 147-170. ISSN: $1139-5737$ 
ciencia desde la pura obsesión teorética, reconociendo sus poderes materiales. La ciencia no es gente que habla con gente de otra gente. Pickering no la concibe como una construcción en términos conceptuales y lingüísticos, sino más bien como una actividad social y material. Al Laboratorio, que es el lugar en donde se produce ciencia, se llega pasando por el Taller, y no sólo por la Academia. Hacer más bien que pensar.

\section{EL CIENTÍFICO COMO SUJETO PERFORMATIVO Y LA CIENCIA COMO CULTURA MATERIAL}

Buscando entender la recíproca co-producción de ciencia, tecnología y sociedad, Pickering (1995) realiza un análisis de la práctica científica real que pretende encajar a un mismo tiempo las dimensiones conceptual, material y social de la ciencia, gracias al uso del idioma performativo. Pickering forja la imagen del «mangle» (rodillo, exprimidor) para dar cuenta del proceso dialéctico de resistencia y acomodación entre el científico y el mundo, a través de las máquinas, los aparatos y los instrumentos. Pickering (1995: 7) apuesta por concebir los científicos como agentes humanos que continuamente actúan en un campo de agencia material. Aparte de construir teorías y modelos, los científicos diseñan máquinas para manipular la realidad.

Se trata de anteponer lo práctico a lo teórico a la hora de concebir al científico. La razón práctica es la razón originaria, y la razón especulativa es su resultante. La racionalidad científica tiene tanto que ver con el cuerpo como con la mente. «Ni la mano sola ni el espíritu abandonado a sí mismo — decía Francis Bacon en Novum Organum (I, 2) - tienen gran potencia; para realizar la obra se requieren instrumentos que tan necesarios son a la inteligencia como a la mano». De hecho, algunos físicos, como Ferrero, Salgado y Sánchez-Gómez (2007) aseveran, con acentuado materialismo, que la racionalidad científica no reside en el cerebro de los científicos ni en su conducta, sino en la propia ciencia en cuanto institución encarnada en la realidad material. No es algo sobreañadido al dominio de la ciencia, sino la misma composición operatoria de los materiales de la ciencia por los sujetos corpóreos llamados científicos. Recogiendo el testigo materialista, Gustavo Bueno (1992: 111) afirmará, como veremos más adelante, que «el homo sapiens es indisociable del homo faber». Aparte de cerebro y laringe, los científicos tienen manos y aparatos a mano. Son, por así decir, las manos - las operaciones quirúrgicas o manuales-, las que construyen racionalmente el mundo.

En cuanto cultura, la multiplicidad de objetos que cae dentro de ese todo complejo llamado ciencia puede ser clasificada bajo tres grandes rúbricas: ideas, signos y cosas (Hacking 1992: 44). Ideas, signos y cosas se vinculan entre sí en la ciencia como los factores de un monomio, de tal modo que si alguno de ellos fuera anulado, ello supondría la anulación del conjunto, es decir de la ciencia. En particular, por tanto, cualquier ciencia ha de incluir necesariamente

EMPIRIA. Revista de Metodología de Ciencias Sociales. N. ${ }^{\circ}$ 18, julio-diciembre, 2009, pp. 147-170. ISSN: 1139-5737 
un estrato fisicalista. Nos encontramos, pues, con la necesidad de desarrollar —como mantiene Norton Wise (2006: 81) — una «epistemología materializada» que reúna el conocimiento teórico (ideas) con el práctico (signos, cosas).

Tarja Knuuttila (2005) ha dado un paso importante en esta dirección al ofrecer una visión material de las teorías y los modelos teóricos, a los que denomina «artefactos epistémicos». Existe una fuerte tendencia a distinguir los objetos teóricos de los objetos materiales en la ciencia. Sin embargo, como advierte Knuuttila, las teorías, que suelen ser presentadas mediante diagramas, dibujos o lenguajes formales y naturales, siempre aparecen materializadas como inscripciones en el papel o en la pantalla. A su vez, los objetos materiales siempre guardan relación con interpretaciones, significados y conocimientos. Esto es válido incluso para cosas que habitualmente son consideradas como simples herramientas, como los instrumentos y los aparatos de laboratorio, a los que Peter Galison (1997) denomina «máquinas epistémicas». Todos los objetos de la cultura científica participan, empero, de esta dimensión material.

\section{MÁS ALLÁ DEL REALISMO PRAGMÁTICO Y EXPERIMENTAL: EL REALISMO MATERIALISTA}

Desde el momento en que consideramos al científico como un sujeto técnico-operatorio y, por tanto, los pesos, los muelles, las balanzas, los metros, los relojes, los dinamómetros, los barómetros, las bombas de vacío, los imanes, los electroscopios, los amperímetros, los telescopios, los microscopios, los aceleradores de partículas y las modernas computadoras como materiales de la ciencia, al igual que los modelos y las teorías, los libros y las revistas, el estudio de la ciencia experimenta un giro de ciento ochenta grados. Para Hacking y Pickering, los filósofos y los sociólogos se han limitado a pensar la ciencia como descripción o representación, pero de lo que se trata es de pensarla como transformación de la realidad. Además, asumir el idioma performativo permite ir más allá, abordar desde nuevas claves los problemas tradicionales de la verdad y la objetividad de la ciencia:

Quiero señalar, primero, que el análisis de la práctica científica y el cambio del idioma representacional por el performativo puede servir para desplazar la problemática tradicional, para transformarla en algo bastante diferente —más interesante, pienso, y menos intratable-. [...] En el idioma performativo, uno no está obligado a discutir la relación entre conocimiento y mundo en términos de correspondencia. En su lugar, uno puede preguntar cómo, en la práctica, las conexiones entre el conocimiento y el mundo se construyen y en qué consisten (Pickering 1995: 179).

Por ejemplo: Pickering (1989 y 1995) intenta resolver la cuestión del realismo científico mediante la propuesta de un realismo pragmático o performativo. El realismo pragmático reclama una visión constructivista de la práctica científica, que

EMPIRIA. Revista de Metodología de Ciencias Sociales. N. ${ }^{\circ}$ 18, julio-diciembre, 2009, pp. 147-170. ISSN: $1139-5737$ 
afecta tanto a las teorías y los modelos matemáticos como a los instrumentos, los aparatos, las máquinas e, incluso, los propios científicos. Pickering busca, de este modo, superar la tradicional dicotomía entre pensar y hacer, entre lo ideal y lo material. El realismo pragmático especifica conexiones no triviales entre el conocimiento y el mundo, que son bastante diferentes de la relación de correspondencia y consisten en la estabilización interactiva de máquinas y conceptos, entre el mundo material y el mundo de la representación (1995: 182).

Por su parte, Hacking (1992: 31) afirma que, aunque el fin de su filosofía es significativamente distinto de la meta que persigue la sociología del conocimiento de Pickering, su realismo experimental «vive felizmente como parte del realismo pragmático». Ambos insisten en la práctica frente a la teoría, en la construcción o intervención frente a la representación. Hacking (1996: 249) sostiene, en efecto, que «los científicos crean los fenómenos que posteriormente se convierten en las piezas centrales de la teoría». Hacking funda la ciencia en la manipulación de la realidad, y concluye: «no es pensar acerca del mundo, sino cambiarlo, lo que al final tiene que hacernos realistas científicos» (1996: 16). A fin de cuentas, como sentencia Allan Franklin (2002), no hay antirrealistas dentro de un laboratorio: los físicos experimentales creen en la realidad de los electrones, los positrones y demás entidades porque han aprendido a manejarlos de diversos modos.

Sin embargo, tanto el realismo pragmático como el realismo experimental presentan ciertas limitaciones insalvables. El realismo pragmático emerge como un marco interpretativo que pretende unificar el carácter fragmentado e inconexo de la literatura existente en el campo de la SCC. Pero, a causa de ello, gran parte de su fuerza se disipa, por cuanto dentro de la SCC los intereses gnoseológicos no son siempre coincidentes ni compatibles. Por poner un ejemplo: cuando Shapin y Schaffer publicaron Leviathan and the Air Pump en 1985, la obra llevaba como subtítulo «Hobbes, Boyle y la vida experimental»; en cambio, cuando ocho años después la obra se tradujo al francés por influjo de Latour, el subtítulo cambió notablemente: «Hobbes y Boyle entre la ciencia y la política». El libro era y no era el mismo. El foco estaba en otra parte. Siguiendo esta estela, múltiples sociólogos de la ciencia han devorado los valores gnoseológicos con valores sociales. Pickering (1984), por ejemplo, argumenta que las razones para aceptar ciertos resultados y no otros son la posible futura utilidad de los mismos, así como su concordancia con las creencias de la comunidad científica. Por consiguiente, Pickering puede calificar de realista su concepción de la ciencia y, a pesar de ello, su concepción puede no tener de realismo más que el nombre. De hecho, Pickering (1995: 183 n. p.) reconoce que muchos colegas le han preguntado por qué la denomina realista. Según él, tanto por motivos retóricos (para no poner en guardia antes de tiempo a quienes no creen que la ciencia sea una construcción social) como porque está en contra del monopolio del realismo por parte de los representacionistas. Pickering (1995: 171) se declara agnóstico frente al realismo de correspondencia, porque la controversia tradicional sobre la correspondencia entre la teoría y el mundo es uno de los tópicos centrales del idio-

EMPIRIA. Revista de Metodología de Ciencias Sociales. N. ${ }^{\circ}$ 18, julio-diciembre, 2009, pp. 147-170. ISSN: $1139-5737$ 
ma representacional que desaparece (supuestamente) al asumir el idioma performativo. Pero Pickering (1995: 192 n. p.) también se declara neutral con respecto al realismo de entidades por el que aboga Hacking, y esto marca los límites gnoseológicos de su enfoque, dado que desatiende la problemática de la verdad de la ciencia a través de la práctica.

Por su parte, el problema con el realismo experimental radica en que no todos los que se encuentran en las filas del NE asumen el bagaje gnoseológico de su líder, de Ian Hacking. En otros términos, como señala Camprubí (2005), no hay uno sino muchos nuevos experimentalismos. No existe unicidad. En efecto, unos - como Galison o Franklin-, que son la mayoría, emplean la doctrina experimentalista como mera metodología o heurística para hacer historia o sociología de la ciencia, mientras que otros — como Hacking y Cartwright- la utilizan como toma de posición con respecto a las controversias tradicionales (verdad científica, realismo científico...). Es decir, se puede ser nuevo experimentalista y, simultáneamente, teoreticista, toda vez que se señalen los contextos tecnológicos de las ciencias pero no se diga nada acerca de su constitución específica. El enfoque experimentalista puede ser solidario del teoreticismo si, pese a subrayar la participación de la técnica en la ciencia, no se es consciente del grado de objetividad que alcanzan las construcciones científicas a través de su génesis operatoria.

En resumidas cuentas, tanto el realismo pragmático como el realismo experimental han llamado la atención sobre el componente material constitutivo de las ciencias, pero ninguno de ellos ha tomado partido por una postura ontológica significativa, con las consecuencias gnoseológicas que de ello se deduzcan. Hacking (1999: 71) defiende que Pickering (1995) es la contribución más materialista a los estudios sociales de la ciencia hasta la fecha; y Hacking (1992: 31) apunta que su tesis es materialista, tanto en su atención al lado material de lo que se hace en ciencia como en su oposición al intelectualismo. Y, sin embargo, ni Pickering ni Hacking han subrayado la importancia del componente material en ciencia con la radicalidad ontológica del realismo materialista de Gustavo Bueno. Últimamente se ha señalado mucho el papel de los aparatos e instrumentos en ciencia, pero sólo Bueno $(1992,182-183)$ ha llegado a afirmar que los aparatos son el lugar de la verdad en la ciencia.

\section{LOS APARATOS COMO CONTEXTOS DETERMINANTES DE LAS CIENCIAS}

Desde el realismo materialista de Gustavo Bueno, las ciencias son antes que nada instituciones sociales cuya función es construir cierta clase de composiciones que llamamos verdades. Ahora bien, la concreción de esta función o trabajo no es, originariamente, escribir libros o artículos. Los productos lingüísticos son sólo un aspecto de la ciencia y si nos atenemos únicamente a él, seremos víctimas del «fetichismo de la mercancía». Del mismo modo que la mú-

EMPIRIA. Revista de Metodología de Ciencias Sociales. N. ${ }^{\circ}$ 18, julio-diciembre, 2009, pp. 147-170. ISSN: 1139-5737 
sica no reside en los pentagramas, puesto que tiene que sonar, las verdades científicas no pueden reducirse, en la línea del teoreticismo, a las proposiciones y las ecuaciones. «La Física no se reduce al Handbuch der Physik: comporta, esencialmente, manipulaciones con objetos físicos, movimientos del cuerpo, fabricación de aparatos» (Bueno 1970: 208). Las ciencias son, pues, más que teorías: comportan arsenales de términos y aparatos. Se trata de desplazar la atención de las cristalizaciones simbólicas hacia el campo operatorio de cada ciencia.

La teoría de la ciencia de Bueno, denominada teoría del cierre categorial, consiste en un constructivismo de signo materialista, porque no se limita al terreno de las construcciones conceptuales y defiende que las operaciones científicas son «quirúrgicas» (manuales) antes que meramente mentales. La intrincación entre técnicas, ciencias y tecnologías es, desde luego, insoslayable. Siguiendo la Tesis II de Marx sobre Feuerbach («El problema de si al pensamiento humano se le puede atribuir una verdad objetiva, no es un problema teórico, sino un problema práctico: es en la práctica donde el hombre tiene que demostrar la verdad, es decir, la realidad y la fuerza, la terrenalidad de su pensamiento»), Bueno va a sostener que la praxis demuestra la verdad de la ciencia: verum est factum. "La ciencia moderna —afirma Bueno (1992: 39)— nos enseña qué significa poseer la verdad de la conexión entre cosas (verum est factum) y qué significa no poseerla, andar a ciegas o por tanteo (en el momento de curarnos de una enfermedad, explotar una bomba atómica o dirigir una astronave a Marte)». Para decirlo radicalmente: la verdad de la Ecuación de Einstein $\mathrm{E}=\mathrm{mc}^{2}$ descansaría en las explosiones atómicas llevadas a cabo por los físicos en los atolones del Pacífico. La capacidad para transformar los micro-, meso- y macro-cosmos determina la fuerza de las verdades que construyen las ciencias.

Para Bueno, las ciencias segregan verdades; pero estas verdades, lejos de describir o representar el mundo, construyen el mundo, lo hacen. Las ciencias no cambian nuestra imagen del mundo, cambian el propio mundo. La estrella polar del realismo materialista es concebir las ciencias como construcciones «con las cosas mismas». Por ejemplo: «son los electrones, los protones y los neutrones (y no sus símbolos o sus funciones de onda) —en tanto, es cierto, están controlados por los físicos en aparatos diversos (tubos de vacío, ciclotrones, etc.) — los que forman parte de la física» (1995: 41). Lógicamente, esta tesis resultará extraña a quien considere los aparatos como algo externo a las ciencias, pero «¿acaso la física no cuenta como contenidos internos suyos a las balanzas de Cavendish, a los planos inclinados, a las cámaras de Wilson o a los pirómetros ópticos?» (1995: 42). Es más, «las fórmulas mismas para que tengan sentido, para que mediante ellas puedan planearse experimentos, suponen los aparatos» $(1982: 373)^{4}$.

\footnotetext{
${ }^{4}$ Hago notar que la solución gnoseológica de Bueno no pasa - como podría presuponerse alarmantemente - por reemplazar las fórmulas por los aparatos y marginar el plano de las teorías en un giro completo (el péndulo yendo al otro lado), sino en comprender el significado de las teorías a través de las prácticas: «las fórmulas suponen los aparatos». Pasamos, pues, del estilo representa-
}

EMPIRIA. Revista de Metodología de Ciencias Sociales. N. ${ }^{\circ}$ 18, julio-diciembre, 2009, pp. 147-170. ISSN: $1139-5737$ 
La ciencia no está, en origen, en los libros sino en los laboratorios, como la física no está en las fórmulas sino en los aparatos. En consecuencia, las leyes de la física no son las Leyes de la Naturaleza, sino las leyes del laboratorio que envuelve a los físicos y, por extensión, del mundo que envuelve al resto de sujetos operatorios (Bueno 1992: 1204). Son leyes objetivas pero, simultáneamente, relativas a los aparatos y las operaciones del físico. Aún más: estas leyes o regularidades no están depositadas en el cielo o en el pensamiento, sino en las construcciones materiales (dispositivos experimentales). En una palabra: en los aparatos. Los aparatos son los «contextos determinantes» de las ciencias. Bueno (1992) llama contextos determinantes a las armaduras materiales que posibilitan las construcciones científicas y, en general, los «cierres categoriales» de las ciencias ${ }^{5}$. Los aparatos constituyen, desde luego, las armaduras experimentales a través de las cuales las teorías se convierten en mecánica o en física, se hacen realidad. Este audaz paso adelante que Bueno da, poniendo de relieve los aparatos, se funda en que «el lugar de la verdad científica es la armadura o contexto determinante en que se constituye» (1992: 182). Por ejemplo: el oxígeno debe ser reconocido como una verdad desde la perspectiva de la Campana de Lavoisier, que - junto al aire que contenía, el óxido rojo de mercurio y las operaciones tecnológicas de los científicos que pusieron a punto el dispositivo- constituyó la armadura del experimento de 1776 y que, a día de hoy, sigue siendo válido aunque pueda ser sofisticado de cara a mejorar su eficiencia. Esta idea también está en Hacking (1992: 30): «Las teorías de las ciencias de laboratorio no son directamente comparables al 'mundo'; las teorías persisten porque son verdad con respecto a fenómenos producidos o hasta creados mediante aparatos en el laboratorio, y que además son medidos mediante instrumentos que nosotros hemos construido» (cursivas mías). «Un instrumento en la ciencia moderna es - por decirlo metafóricamente con Bachelard- realmente un teorema».

Pero conviene recalcar que la tesis de que la verdad científica compromete, inmediatamente, a los aparatos y no al mundo o al sujeto (salvo de un modo mediato) no es inocua. Efectivamente, conlleva aparejada la aceptación de que cada ciencia trabaja una parcela de realidad acotada por el alcance y la capacidad de sus aparatos, y que es dentro de ese dominio donde son válidos los teoremas construidos por los científicos. De hecho, cada grupo de leyes físicas va siempre referido a un dominio de validez, que queda determinado por la clase de fenó-

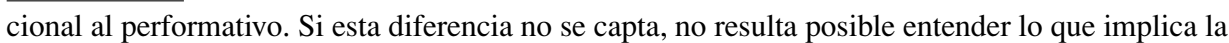
nueva teoría materialista o pragmatista de la verdad científica, que desborda el marco lingüístico (el protocolo carnapiano o la observación de una mente pura), para comprender las verdades que construyen las ciencias más bien como ejercicios que como representaciones (la verdad estaría en el experimento).

5 Bueno (1992) llama «cierre categorial» al resultado de la empresa científica: cuando las operaciones de los científicos determinan un campo de términos, objetos, instrumentos, aparatos y relaciones diferenciado del resto de campos científicos, se produce el cierre de una nueva categoría y, con ello, la cristalización de una nueva ciencia.

EMPIRIA. Revista de Metodología de Ciencias Sociales. N. ${ }^{\circ}$ 18, julio-diciembre, 2009, pp. 147-170. ISSN: $1139-5737$ 
menos reproducibles con los aparatos accesibles y por el poder resolutivo de los instrumentos de medida (que marcan límites relativos, digamos, a la masa y la velocidad de los sistemas).

Generalizando: sin aparatos no hay verdades, y sin verdades no hay ciencia. Una ciencia sin aparatos ni verdades es como un círculo cuadrado. Estamos ante el criterio demarcativo de los aparatos: «No cabe hablar, desde luego, de una ciencia sin aparatos, salvo que se mantenga una concepción 'mentalista' o 'lingüística' de la ciencia» (Bueno 1992: 683). Ahora bien, a la luz del criterio de los aparatos, la franja de verdad de las ciencias teóricas y observacionales es mucho más estrecha que la de las ciencias experimentales o de laboratorio. Al carecer de aparatos adecuados que permitan las construcciones por medio de las cuales los científicos interfieren con el curso de la naturaleza, la franja de verdad de la cosmología, de la teoría del big-bang o de la teoría de cuerdas es mucho más estrecha de lo que a priori se antoja (Hacking 1989: 578; Bueno 1992: 1195). Cuando miramos la realidad bajo el farol de la ciencia, más que penetrar en el fondo de ella como si estuviera escrita en caracteres matemáticos, estamos aprehendiendo el funcionamiento real de las cosas bajo nuestras construcciones; pero, a día de hoy, no manejamos construcciones que nos permitan interaccionar con singularidades cósmicas o con cuerdas. Curiosamente, algunos físicos comienzan a reparar en el callejón sin salida de la teoría de cuerdas: para Penrose (2006), Smolin (2007) o Ynduráin (2007) es física-ficción, llena de fantasías especulativas. Además, como contraprueba del criterio de los aparatos, puede aducirse que es capaz de explicar por qué la Alemania Nazi no logró fabricar la Bomba Atómica. No es que faltaran cabezas, es que faltaban aparatos. En sus diarios, Albert Speer, Ministro de Armamento del III Reich, cuenta que el gran Werner Heisenberg le dijo en una conversación privada que iba a ser muy difícil fabricar la bomba porque la industria alemana estaba muy menguada. En efecto, como recoge Sánchez Ron (2007: 681), en 1938, justo antes del comienzo de la contienda, Estados Unidos ya disponía de 8 aceleradores de partículas y proyectaba otros tantos, Inglaterra disponía de 2 y, sin embargo, Alemania aún pugnaba por construir el primero.

Sólo resta una pregunta: ¿por qué el papel sociológico (Pickering), epistemológico (Hacking) y ontológico (Bueno) de los aparatos ha tardado tanto tiempo en ser señalado? ¿Por qué la relevancia gnoseológica de los aparatos no ha sido subrayada hasta la SCC, el NE o el Materialismo Filosófico? Porque a los aparatos en ciencia les ha ocurrido lo que al término medio del silogismo, que cae en el olvido tras ser empleado. En efecto, de igual manera que $B$ desaparece en el silogismo $« A \rightarrow B$ y $B \rightarrow C$, ergo $A \rightarrow C »$, los aparatos han desaparecido en el silogismo gnoseológico: «Nosotros manejamos aparatos que, a su vez, manejan entes reales; luego nosotros manejamos entes reales»... y se olvida la imprescindible mediación de los aparatos. Precisamente, este olvido es, como a continuación vamos a comprobar, especialmente gravoso en física cuántica. Los físicos cuánticos no manipulan partículas subatómicas inmediatamente, lo hacen siempre de modo mediato. Nuestro trato con los sistemas cuánticos viene

EMPIRIA. Revista de Metodología de Ciencias Sociales. N. ${ }^{\circ}$ 18, julio-diciembre, 2009, pp. 147-170. ISSN: 1139-5737 
necesariamente mediado por los aparatos (aceleradores lineales o circulares, cámaras Wilson, etc.). No hay tanta diferencia entre un físico clásico y un físico cuántico, porque ambos se ocupan de procesos —uno, macroscópicos; otro, microscópicos - a través de dispositivos e ingenios mesocósmicos. La física cuántica es, según vamos a comprobar, una red de teorías, modelos, aparatos y realidades interconectadas.

\section{EL CASO DE LA FÍSICA CUÁNTICA (I): LOS APARATOS COMO «CAJAS DE LAS ESENCIAS CUÁNTICAS»}

En la imagen tradicional de la física cuántica, todo está subordinado a la teoría. La actividad científica por excelencia es la especulación teórica, y los experimentos sólo se diseñan para verificar o falsar teorías. Pero, así como la teoría es un factor en el diseño experimental, la práctica interviene en la construcción de teorías. No sólo para contrastarlas, sino también para llenar los espacios en blanco. Muchas veces las teorías se escriben por medio de los aparatos experimentales. Desde el momento en que consideramos los aceleradores de partículas, las cámaras de niebla o los espectroscopios como materiales de la física cuántica, al igual que los modelos y las teorías, la concepción de la física cuántica cambia radicalmente. La física cuántica no es tanto cosa de libros (de saber) como de laboratorios (de hacer). Desde los postulados de la SCC y del NE, la física cuántica aparece como una construcción material con los objetos cuánticos en vez de como una representación ideal de esos objetos, cuya verdad consiste en un ejercicio encarnado en la realidad antes que en una representación de esa realidad (reacciones nucleares, bombas atómicas, láseres, superconductores...).

Históricamente, las labores científicas de Robert Millikan y Ernest Rutherford ilustran esta fértil dialéctica entre teoría y experimentación, en que la experimentación es la continuación de la teoría por otros medios (y sin perjuicio de que la teorización sea, como dice Van Fraassen (1996), la continuación del experimento por otros medios). Consideremos, en primer lugar, el experimento de la gota de aceite de Millikan para medir la carga del electrón. Hacia 1909, Robert Millikan construyó un aparato que constaba de un atomizador que soltaba una lluvia de diminutas gotas de aceite que, tras ser ionizadas, se dejaban suspendidas en el aire de una cámara compensando la fuerza gravitatoria con una fuerza eléctrica (ver Figura 1). Jugando con el peso de las gotitas y las baterías eléctricas, Millikan logró dejar flotando muchísimas gotitas. Y repitiendo muchas veces el experimento, concluyó que todas las gotas de aceite tenían una carga eléctrica múltiplo de un número muy pequeño: la carga del electrón.

Consideremos, en segundo lugar, el experimento de Rutherford en el descubrimiento del núcleo atómico. En torno a 1910, Ernest Rutherford disponía de un magnífico laboratorio en Manchester, cuyo parecido con el taller electricista de

EMPIRIA. Revista de Metodología de Ciencias Sociales. N. ${ }^{\circ}$ 18, julio-diciembre, 2009, pp. 147-170. ISSN: 1139-5737 


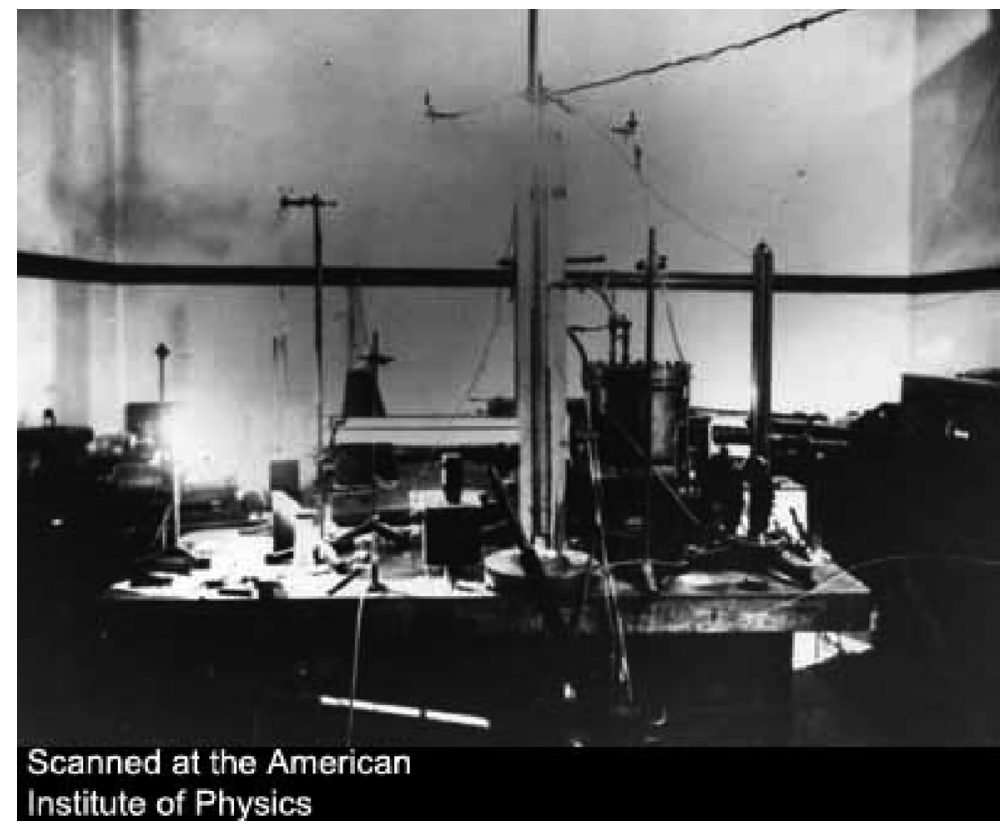

Figura 1. Aparato de Millikan (AIP Emilio Segre Photo Archive).

un chapuzas de barrio es increíble (ver Figura 2). Junto a dos aventajados aprendices, Geiger y Marsden, Rutherford estaba dedicado a bombardear finas láminas de oro o platino con un haz de partículas alfa. La elección de metales tan caros no era casualidad: Rutherford, como los artesanos orfebres, sabía que sólo estos metales pueden cortarse delgadísimamente. La gran mayoría de partículas alfa (que son átomos de helio doblemente ionizados, muy masivos y rápidos) atravesaban la lámina y salían dispersadas con un pequeño ángulo de inclinación. Pero una cantidad no despreciable de ellas (en promedio, una de cada ocho mil) rebotaban frontalmente y salían despedidas hacia atrás. Según sus propias palabras, «era como si se disparara un obús naval de buen calibre sobre una hoja de papel y saliera rebotado». Enseguida, Rutherford concluyó que este fenómeno tenía que ser consecuencia de la colisión de la partícula alfa (de carga positiva) con algo también muy masivo y cargado positivamente (para que hubiera repulsión). Esto certificó la existencia de un núcleo atómico y llevó a Rutherford a la construcción de su modelo planetario de átomo. El átomo de Rutherford constaba de un núcleo (concentrado, cargado positivamente y en que estaban confinados los protones) y de una corteza (amplia, en que orbitaban los diminutos electrones).

Los notables avances de Millikan y Rutherford ponen de relieve la importancia de los aparatos en la física cuántica. Pero, ¿cuál es su estatuto ontológico? A resultas de lo dicho, los aparatos cuánticos tienen reservado un lugar muy es-

EMPIRIA. Revista de Metodología de Ciencias Sociales. N. ${ }^{\circ}$ 18, julio-diciembre, 2009, pp. 147-170. ISSN: $1139-5737$ 
una idea de Bueno (1992). De igual manera que desde un punto de vista sintáctico los objetos lógico-matemáticos se clasifican en términos, funciones o relaciones, los aparatos pueden clasificarse en términos, operadores o relatores. Así, por ejemplo, una pesa de 1 kilogramo funciona como un término. Un microscopio no es, entonces, una prolongación auxiliar del ojo, cuanto un operador objetivo que transforma configuraciones microcósmicas en configuraciones mesocósmicas. Y una balanza no es un instrumento de comparación al servicio del sujeto, sino un relator interpuesto él mismo entre contenidos del campo ${ }^{6}$. Por consiguiente:

a) Términos. Serían aparatos que funcionan como términos en física cuántica las muestras radiactivas, el agua pesada, los materiales de centelleo y fulguración, las emulsiones fotográficas para trazas o huellas e, incluso, los globos sonda o los generadores electrostáticos de Van der Graaff, que son parte de otros dispositivos o aparatos más grandes.

b) Operadores. Serían operadores tanto los aparatos clásicos para manipular partículas (los tubos de Geissler, de Coolidge, de rayos catódicos...) como los modernos aceleradores de partículas. Los famosos aceleradores lineales y circulares - ciclotrones - , construidos respectivamente por Cockcroft y Walton en 1930 y por Lawrence en 1932, consisten en conductos o anillos, de varios kilómetros de longitud, donde las partículas se aceleran, bajo la acción de campos electromagnéticos, hasta velocidades cercanas a la de la luz, para luego hacerlas golpear diversos blancos, como placas de plomo u otras partículas (por ejemplo, un chorro de electrones contra un chorro de positrones). A partir de los años 50, el sistema acelerador-blanco-detector se vio notablemente mejorado al quedar capacitado para producir altísimas energías. Son los cosmotrones (Brookhaven), bevatrones (Berkeley) y tevatrones (Fermilab), hasta llegar al nuevo Gran Colisionador de Hadrones (LHC) del CERN, conocido como la «máquina del big-bang».

c) Relatores. Distinguimos cuatro tipos de aparatos cuánticos encaminados a producir relaciones medibles. En primer lugar, los espectroscopios y espectrómetros, surgidos a finales del XIX de la composición de tubos de descarga, prismas y placas fotográficas que registran los espectros atómicos. En segundo lugar, los espectrógrafos de masas, debidos a Francis Aston en 1919, y que son cámaras selladas en que se introducen átomos que, tras ionizarlos y someterlos a un campo magnético que los obliga a desplazarse en trayectorias curvas, se separan a consecuencia de la distinta inercia, que depende de sus masas diferentes, lo que permite separar los isótopos de un elemento. En tercer lugar, los registradores de trazas: las cámaras de niebla (Wilson), las cámaras de burbujas (Glaser) y las cámaras de chispas (Keiffel). Las cámaras de niebla, inventadas por Charles Thomson Rees Wilson en 1911, no son sino un recipiente con

${ }^{6}$ A veces, efectivamente, un mismo aparato puede actuar en distintos contextos como término, operador o relator (agradezco a David Alvargonzález que llamara mi atención sobre este punto).

EMPIRIA. Revista de Metodología de Ciencias Sociales. N. ${ }^{\circ}$ 18, julio-diciembre, 2009, pp. 147-170. ISSN: 1139-5737 


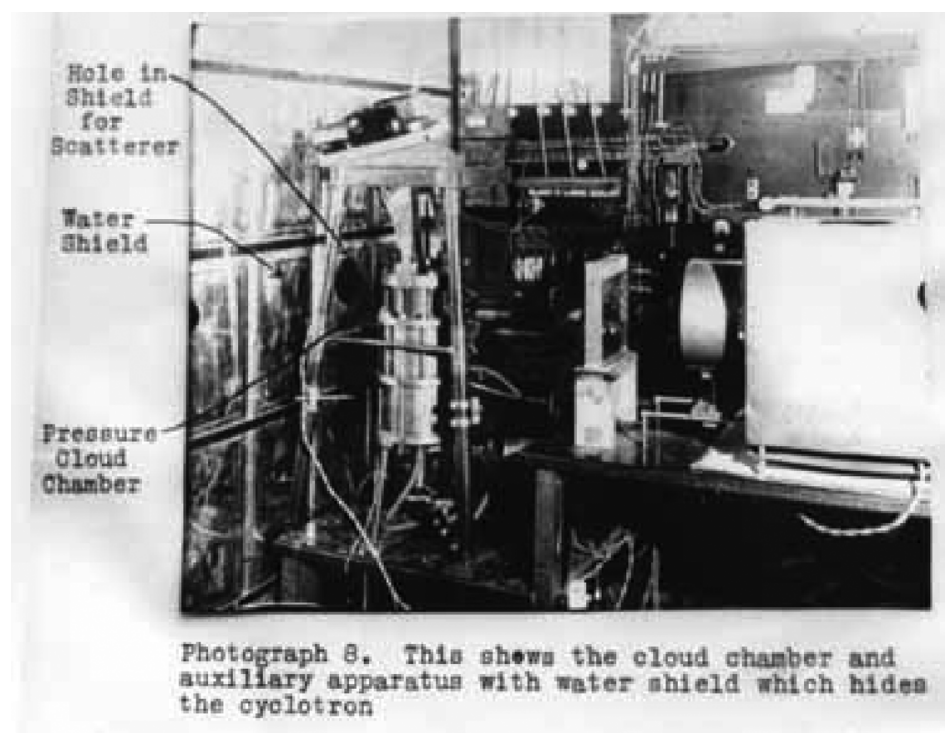

Scanned at the American Institute of Physics

Figura 3. Cámara de niebla conectada a un ciclotrón (AIP Emilio Segre Photo Archive).

gas saturado de humedad que, al ponerse en contacto con una sustancia radioactiva que emite partículas, permite determinar sus trayectorias siguiendo la estela de diminutas gotitas de agua condensadas que dejan al pasar (el vapor condensa alrededor de la partícula viajera). El estudio de la forma, la longitud, la anchura y la curvatura ante campos de las trazas permite extraer mucha información (por ejemplo, los electrones determinan líneas tenues por su poca masa pero largas por su gran velocidad, mientras que los rastros que dejan los protones son más gruesos y cortos). Hacia 1953, Donald Arthur Glaser mejoró la cámara de Wilson construyendo la cámara de burbujas (que, en lugar de aire cargado de humedad, contiene hidrógeno líquido a punto de entrar en ebullición, lo que deja un rastro de burbujas en vez de gotas). También hay cámaras de ionización o de chispas que contienen gases cuyos átomos se ionizan al paso de las partículas. Y, por último, en cuarto lugar, los contadores: tanto los contadores por descarga (Geiger-Müller) como los contadores por centelleo (Curron-Baker). Por ejemplo, un contador geiger consiste en un tubo dotado de un intenso campo eléctrico de manera que cada partícula que penetra da lugar a un impulso que queda registrado (Borovói 1985). 


\section{EL CASO DE LA FÍSICA CUÁNTICA (Y II): EL MUNDO CUÁNTICO COMO «HIPERREALIDAD»}

Ya hemos intentado dilucidar el estatuto ontológico de los aparatos, pero ¿cuál es el estatuto ontológico de los átomos, las partículas $\alpha$ o los electrones? Las partículas elementales, ¿son descubiertas o construidas? Nos encontramos aquí con la cuestión de la materialidad que cabe atribuir a los objetos cuánticos. Desde la SCC, siguiendo la estela de Collins, Woolgar y Latour, Pickering (1982 y 1984) ha defendido que, por ejemplo, los quarks son una construcción social. Hacking (1999: cap. 1) aconseja que siempre que algún sociólogo afirme que $\mathrm{X}$ es una construcción social, no hay que preguntarle por qué $\mathrm{X}$ es una construcción social sino qué es X. En verdad, según Hacking (1999: 30), Pickering no defiende que los quarks —que, de acuerdo con el modelo estándar, son los ladrillos básicos del universo - son una construcción social, sino más bien que el concepto de quark lo es. Este concepto no sería una solución inevitable y, claro, la física podría haber tomado otra ruta. Es muy posible que diversas teorías puedan ser compatibles al tiempo con el mundo, pero que puedan no quiere decir que deban. Lo importante es que el mundo determina qué clase de aparatos y qué clase de fenómenos podemos construir.

A la hora de examinar el constructivismo de un sociólogo del conocimiento científico, Hacking (1999: cap. 3) propone contrastar su posición con respecto a tres puntos críticos: 1) la contingencia (es decir, la idea de que la ciencia no está predeterminada a seguir un único camino), 2) el nominalismo (e. d. el antirrealismo con respecto a las entidades teóricas, como los quarks o el electrón), y 3) las explicaciones externas de la estabilidad (la apelación a valores sociales en lugar de epistémicos para justificar el éxito en ciencia). A diferencia del de Pickering y sus colegas, el constructivismo de Bueno (1992) es de corte materialista, lo que conjura cualquier asomo de relativismo. La cuestión es, a saber: ¿cómo hacer compatibles constructivismo y materialismo? ¿Es posible compatibilizar la idea de construcción de la realidad con la idea de verdad material, es decir, 1) con la negación de 2) y 3)?

Si no se puede conocer sin transformar, la ciencia no describe ni representa la realidad sino que la construye: hace el mundo, lo amplía. Y si la empresa científica es el propio mundo haciéndose, la física cuántica es el propio mundo cuántico haciéndose. La física cuántica va conformando o constituyendo, según progresa, una especie de «hiperrealidad», una suerte de ampliación de la realidad, que en su caso afecta a partes de la realidad que no están dadas a escala corporal pero que están conectadas con ella. Es inopinable que, a día de hoy, los fenómenos atómicos, nucleares o cuánticos interesantes son creados en máquinas aceleradoras, determinando un mundo fabricado por los físicos en el laboratorio. De hecho, se piensa a menudo que no existen las partículas fundamentales, porque su determinación no tiene más límite que el que viene impuesto por la tecnología en la construcción de aceleradores. Por ejemplo: múltiples partículas pesadas del modelo estándar (nuevos bariones, constituidos por tres quarks,

EMPIRIA. Revista de Metodología de Ciencias Sociales. N. ${ }^{o}$ 18, julio-diciembre, 2009, pp. 147-170. ISSN: 1139-5737 
uno de ellos belleza) sólo se han observado en el universo actual en las colisiones de los aceleradores de partículas. En resumen, para Bueno (1992), el mundo cuántico - las nuevas partículas cuánticas — - es una hiperrealidad, una ampliación de la realidad, construida por los físicos operando con grandes máquinas, que a su vez se nos aparecen como las cajas de las esencias cuánticas.

Pero, atención, este constructivismo ligado a los aparatos no se desliza por la pendiente del relativismo, por cuanto defiende la objetividad de las partículas dado que son «invariantes» bajo distintos aparatos. Por ejemplo: el electrón aparece en los procesos de descarga en tubos con gases distintos. La creencia en la existencia de los electrones se sustenta en que, empleando múltiples aparatos, hemos determinado su masa, carga, espín... detectándolos como realidades y, además, hemos sido capaces de emplearlos como herramientas para interferir con otras entidades naturales. Para Hacking (1983), los científicos creen en la realidad de los electrones porque han logrado construir diversos aparatos que los utilizan y manipulan para interferir en la naturaleza. Teniendo en mente el dispositivo experimental conocido como Peggy II, del acelerador de partículas de la Universidad de Stanford, que consiste en una fuente de electrones polarizados, Hacking (1983: 23) afirma: «if you can spray them, then they are real». Pero, como apunta Franklin (1997), los físicos creen en la existencia de los electrones desde mucho antes. El experimento de los rayos catódicos de Thomson y el experimento de la gota de aceite de Millikan demostraron prácticamente la realidad del electrón. Así lo expresaba Robert Millikan en 1924:

Un prominente literato se refería recientemente al electrón como «sólo la última hipótesis científica, que se convertirá en el abracadabra de mañana». [...] La prueba más directa e inambigua de la existencia del electrón se encontrará en el experimento que por conveniencia llamaré «el experimento de la gota de aceite». [...] El éxito del experimento realizado en 1909 se debió completamente al diseño del aparato.

En general, creemos en la existencia de los átomos porque: en primer lugar, como dijera Poincaré, «los átomos son una realidad... desde que los sabemos contar», gracias al experimento de Perrin en 1908; en segundo lugar, por los experimentos de Laue sobre las estructuras atómicas cristalinas; en tercer lugar, por los experimentos en cámaras de niebla, pantallas de detección por centelleo y tubos contadores por zumbido; y en cuarto lugar, para no seguir, por el manejo de átomos gracias a los nuevos microscopios. En efecto, como es bien sabido, los microscopios usuales son los microscopios ópticos, inventados a principios del siglo XVII, pero la instalación de sucesivas lentes amplificadoras no mejora su poder de resolución más allá de cierto límite que muy pronto se alcanza. En

7 Cuya identificación es, naturalmente, una tarea compleja y por lo tanto contingente (basta recordar las enseñanzas del éter o del calórico). Su carácter real se determina, en cualquier caso, a posteriori.

EMPIRIA. Revista de Metodología de Ciencias Sociales. N. ${ }^{\circ}$ 18, julio-diciembre, 2009, pp. 147-170. ISSN: 1139-5737 
1936, Ernst Ruska construyó el primer microscopio electrónico, siguiendo la idea del príncipe Louis de Broglie de que los electrones en movimiento se comportan como ondas de longitud muy corta que es posible enfocar para «observar» lo más pequeño. El microcopio electrónico amplió la realidad ya ampliada por el microscopio óptico, al permitirnos trabajar con aquellas partes de la realidad que la luz visible no iluminaba (ésta tiene una longitud de onda muy grande que hace indetectables los objetos que se ocultan bajo su cresta). Desgraciadamente, ni siquiera estos potentes microscopios eran capaces de permitirnos detectar átomos. Pero, recientemente, en torno a 1986, Gerd Binnig y Heinz Rohrer, del Laboratorio de IBM de Zurich, construyeron el microscopio electrónico de barrido de efecto túnel (STM), que permite analizar superficies a escala nanométrica y manipular átomos de uno en uno, hasta el punto de haberse llegado a escribir las siglas de IBM empleando 35 átomos de xenón. La física cuántica construye múltiples cosas que no están dadas en la naturaleza, pero que son materiales y, por ende, reales.

\section{UNA «NUEVA» HISTORIA DE LA CIENCIA Y DE LAS REVOLUCIONES CIENTÍFICAS}

Acabamos de ver cómo se concreta la doctrina experimentalista en la historia de la física cuántica, pero ¿qué consecuencias se desprenden para la historia de la ciencia en general? A nuestro entender ésta es la principal: la historia de la ciencia es, en gran medida, la historia de los contextos determinantes, de los aparatos. Lo que sirve de guía heurística para realizar toda una reconstrucción de, por ejemplo, la historia de la física. A modo de ilustración: pasa por ser un tópico que los filósofos naturales del XVI y XVII descubren el «método científico», pero ¿acaso no lo usaban ya los astrónomos geómetras? ¿En qué se diferencia el proceder de Hiparco o Ptolomeo del de Clairaut o Leverrier? ¿Y el de Pitágoras o Arquímedes del de Galileo o Newton? La respuesta experimentalista es que no hay que buscar la esencia de la «Revolución Científica» en el espíritu o el método científicos, sino en los contextos determinantes. Las grandes etapas de la historia de cada ciencia habría que referirlas a las transformaciones en los equipos de aparatos (Bueno 1992: 686). Pitágoras y Arquímedes experimentaban con cuerdas vibrantes, clepsidras, palancas y poleas; en cambio, Galileo y Newton lo hacían con planos inclinados, péndulos, telescopios y prismas. Ésta es la sutil diferencia.

La «Nueva Ciencia» se impuso gracias a un nuevo arsenal de aparatos. Hacia finales del XVI, el instrumental de las ciencias físicas se reducía a media docena de aparatos (cuadrantes, escalas angulares y demás instrumentos de observación astronómica); pero los siguientes cien años conocen una explosión instrumental en los laboratorios amateurs: microscopios, telescopios, barómetros, termómetros, bombas de aire y demás artilugios. Como señala Kuhn (1993: 69) en «La tradición matemática y la tradición experimental en el desarrollo de

EMPIRIA. Revista de Metodología de Ciencias Sociales. N. ${ }^{\circ}$ 18, julio-diciembre, 2009, pp. 147-170. ISSN: $1139-5737$ 
la física»: «la física se había vuelto instrumentalista». Nombres como los de Galileo, Boyle, Hooke o Newton contrastan con los de Copérnico, Descartes o Pascal. El filósofo natural no sólo sabía geometría euclídea, manejaba con soltura las técnicas artesanales. Aparte de lector de Euclides y Arquímedes, profesor de matemática y artillería, Galileo fue un ensayador, un experimentador que destacó por los inventos que construyó: telescopios refractores, sectores, termoscopios y otros cachivaches. Galileo joven pasó más tiempo en los arsenales de Venecia, entre grúas y cabrestantes, que en la Universidad. Además, al igual que Galileo, Newton también destacó como inventor. Construyó, por ejemplo, el primer telescopio reflector, que no usaba lentes sino espejos, y experimentó con discos y prismas colocados en sorprendentes disposiciones. Desde luego, Salviati arrinconó a Simplicio; pero no con sus mismas armas sino con los nuevos aparatos, como el telescopio, el plano inclinado y el péndulo.

Además, desde la perspectiva experimentalista, la física relativista y la física cuántica no refutaron o falsaron - como suele decirse desde el más puro teoreticismo - la física clásica, sino que sólo la reemplazaron dentro de la parcela de realidad que contiene aparatos como aceleradores de partículas, radiotelescopios, interferómetros, espectrómetros... El progresivo aumento de la precisión instrumental posibilitó experimentos cada vez a velocidades más grandes y a escalas más pequeñas, y la inclusión de nuevos operadores y relatores fue la gota que colmó el vaso de la física clásica. La revolución relativista se produjo a consecuencia de los fenómenos electrodinámicos construidos sobre interferómetros, y la revolución cuántica se fraguó en los fenómenos atómicos construidos con la ayuda de tubos de vacío y espectroscopios. En palabras de Pickering (1995: 189): «Las teorías de la física antigua están asociadas al mundo vía las acciones de un cierto conjunto de máquinas; las teorías de la nueva física se asocian a otro conjunto disjunto». Pero la física clásica sigue viva, todavía se la trabaja y moderniza, porque permite resolver muchos problemas en una aproximación óptima (ningún científico calcula una trayectoria de balística empleando las ecuaciones de la relatividad general). Es decir, sólo de forma harto caprichosa puede negarse que sigue siendo verdad para sistemas experimentales cuya velocidad no se aproxime a la de la luz o cuya masa no sea excesivamente pequeña. Es en este sentido en el que puede hablarse de verdades dentro de sus propios contextos (determinantes).

Más aún, desde la perspectiva experimentalista, la historia de la ciencia precisa de esquemas de transformación y conservación mejor que de revolución. Ni fijismo ni catastrofismo, sino evolucionismo. No hay ciencias revolucionarias, como tampoco hay ciencias reaccionarias. Hay ciencias en continua reforma. El cambio en ciencia es la regla, pero las ciencias posteriores reconstruyen las verdades de las ciencias anteriores (y si no, es que no eran verdad). La marcha de las ciencias se produce sin saltos, con continuidad (aunque continuidad no quiere decir suavidad). Las ciencias posteriores reutilizan — como sugiere Bueno $(1992,1262)$ - los materiales de las ciencias anteriores, a la manera como los sillares o arcos de un edificio destruido se emplean en la construcción del nuevo.

EMPIRIA. Revista de Metodología de Ciencias Sociales. N. ${ }^{o}$ 18, julio-diciembre, 2009, pp. 147-170. ISSN: 1139-5737 
La razón es que los instrumentos y aparatos científicos no pueden cambiar de teorías. Ciertamente, incorporan teorías (de otro modo no tendríamos ni idea del significado de su acción), pero crean una relación invariante entre sus operaciones y el mundo. Algo que hasta el propio Kuhn reconoce, como demuestra la cita que encabeza este artículo. A pesar del extendido entusiasmo en la refutación y las revoluciones teóricas, Hacking (1992: 29) defiende que las ciencias de laboratorio conducen a un conocimiento, un instrumental y unas prácticas persistentes y estables; y añade: «la ciencia de laboratorio estable surge cuando las teorías y el equipo de laboratorio evolucionan de tal modo que se acoplan unas con otros y resultan mutuamente autojustificativos» (1992: 56). Por ejemplo: Hacking (1984: 118) observa que, desde el descubrimiento del efecto fotoeléctrico, se han sucedido múltiples explicaciones, como la einsteiniana del fotón, pero que, pase lo que pase con esta última, que es la que hoy aceptamos, «las puertas de los supermercados (que dependen del efecto fotoeléctrico para su funcionamiento) continuarán funcionando». Si un instrumento da una lectura de X cuando se compone con un fenómeno, continuará mostrando la misma lectura tras un cambio de teoría, aún cuando pueda ser que se considere que ahora dice algo distinto (Ackermann 1985: 33). La persistencia de los resultados experimentales suministra continuidad a través de los cambios conceptuales (Galison 1987; Franklin 2002). Los aparatos son, pues, más robustos que plásticos.

\section{CONCLUSIÓN}

Tras presentar los tres componentes del giro «praxiológico» en los estudios de la ciencia $(\S 1)$, formulamos las tesis fundamentales de la SCC y del NE (§2-3), que apostaban por un enfoque material de las ciencias. Ahora bien, tomar un enfoque material invitaba o conducía a un planteamiento materialista de la ciencia: la función de la ciencia, en cuanto institución social, no sería conocer sino hacer el mundo (\$4-5). Después, asumimos este enfoque a fin de pensar la física cuántica a través de su historia (\$6-7). Y, finalmente, hemos estudiado cómo la visión experimentalista se concreta en una nueva historiografía de la ciencia $(\$ 8)$.

Conocer la realidad (mediante la ciencia) no es otra cosa que hacer la realidad, porque los aparatos científicos son productores de realidades, constructores de mundo. Obviamente, la concepción de la ciencia de Pickering, Hacking o Bueno se encuentra directamente en las antípodas de la imagen teorética tradicional. No en vano, como sabía Ortega y Gasset (1979: 34), «no puede haber nada más contrario a lo que es conocer la Realidad que hacer la Realidad». Pero el poder de la ciencia es tangible y, por decirlo telegráficamente, la transformación del mundo mediante los aparatos es, de facto, la prueba de su verdad y objetividad. En resumidas cuentas, la cuestión no sería tanto «¿Cómo hacer ciencia con aparatos?» sino «¡Cómo no hacer ciencia con aparatos!».

EMPIRIA. Revista de Metodología de Ciencias Sociales. N. ${ }^{\circ}$ 18, julio-diciembre, 2009, pp. 147-170. ISSN: 1139-5737 


\section{BIBLIOGRAFÍA}

ACKeRmann, Robert J. (1985): Data, Instruments and Theory, Princeton, Princeton UP.

- (1989): «The New Experimentalism», British Journal for the Philosophy of Science, 40, pp. 185-190.

BorovóI, A. (1985): Cómo se registran las partículas, URSS, MIR.

BuEno, Gustavo (1970): El papel de la filosofía en el conjunto del saber, Madrid, Ciencia Nueva.

- (1982): «El cierre categorial aplicado a las ciencias físico-químicas», en Actas del I Congreso de Teoría y Metodología de las Ciencias, Oviedo, Pentalfa, pp. 101-164.

- (1992): Teoría del cierre categorial (5 volúmenes), Oviedo, Pentalfa.

- (1995): ¿Qué es la ciencia?, Oviedo, Pentalfa.

CAMPRUBí, Lino (2005): «Experimentalismo y Gnoseología, a propósito de un libro de Galison», El Catoblepas, 42, p. 24.

CARTwright, Nancy (1983): How the Laws of Physics Lie, Oxford, Oxford UP.

FERREIRós, José y ORDÓÑEZ, Javier (2002): «Hacia una filosofía de la experimentación», Crítica, 102, pp. 47-86.

Ferrero, Miguel; Salgado, David y Sánchez-Gómez, José L. (2007): «Física cuántica y orden racional», Revista Iberoamericana de Física, 3/1, pp. 11-16.

FRANKLIN, Allan (1986): The Neglect of Experiment, Cambridge, Cambridge UP.

- (1989): «The Epistemology of Experiment», en D. Gooding, T. Pinch y S. Shaffer (eds.), The Uses of Experiment, Cambridge, Cambridge UP, pp. 437-460.

- (1997): «Are There Really Electrons? Experiment and Reality», Physics Today, 50/10, pp. 26-33.

- (2002): «Física y experimentación», Theoría, XVII/44, pp. 221-242.

Galison, Peter (1987): How the experiments end, Chicago, Chicago UP.

- (1997): Image and Logic: A material culture of microphysics, Chicago, Chicago UP.

HACKING, Ian (1983): Representing and Intervening, Cambridge, Cambridge UP.

- (1984): «Five Parables», en Philosophy in History, Cambridge, Cambridge UP, pp. $117-126$.

- (1989): «Extragalactic Reality: The Case of Gravitational Lensing», Philosophy of Science, 56, pp. 555-581.

- (1992): «The Self-Vindication of the Laboratory Sciences», en Andrew Pickering (ed.), Science as Practice and Culture, Chicago, Chicago UP, pp. 29-64.

- (1996): Representar e Intervenir, Barcelona, Paidós.

- (1999): The Social Construction of What?, Cambridge, Harvard UP.

Heilbron, John Lewis (1979): Electricity in the $17^{\text {th }}$ and $18^{\text {th }}$ Centuries. A Study in Early Modern Physics, Nueva York, Dover.

- (1999): The Sun in the Church: Cathedrals as Solar Observatories, Nueva York, Dover.

HeILBRON, John L. y SEIDEL, Robert W. (1989): Lawrence and his Laboratory: A History of the Lawrence Berkeley Laboratory, Chicago, Chicago UP.

KnuUtTila, Tarja (2005): «Models, Representation, and Mediation», Philosophy of Science, 72, pp. 1260-1271.

Kunn, T. S. (1962): The Structure of Scientific Revolutions, Chicago, Chicago UP.

- (1993): La tensión esencial, Madrid, FCE.

MADRID CASADO, Carlos (2005): «A vueltas con Ortega, la Física y Einstein», Revista de Occidente, 294, pp. 5-20. 
- (2006): «El Nuevo Experimentalismo en España: entre Gustavo Bueno e Ian Hacking», Contrastes. Revista Internacional de Filosofía, XI, pp. 153-169.

- (2008): «Filosofía de la Física. El Cierre de la Mecánica Cuántica», El Basilisco, 39, pp. 67-112.

ORTEGA Y GASSET, José (1979): La idea de principio en Leibniz, Madrid, Alianza.

PenRose, Roger (2006): La nueva mente del Emperador, Barcelona, DeBols!llo.

Pickering, Andrew (1982): «Elementary Particles: Discovered or Constructed?», en W. P. Trower \& G. Bellini (eds.), Physics in Collision: High-Energy eelep/pp Interactions, Nueva York, Plenum, pp. 439-48.

- (1984): Constructing Quarks: A Sociological History of Particle Physics, Chicago, Chicago UP.

- (1989): «Living in the Material World: On Realism and Experimental Practice», en D. Gooding, T. Pinch y S. Shaffer (eds.), The Uses of Experiment, Cambridge, Cambridge UP, pp. 275-297.

- (1992): «From Science as Knowledge to Science as Practice», en A. Pickering (ed.), Science as Practice and Culture, Chicago, Chicago UP, pp. 1-26.

- (1995): The Mangle of Practice: Time, Agency and Science, Chicago, Chicago UP.

SÁnCHEZ Ron, José Manuel (2007): El poder de la ciencia, Barcelona, Crítica.

Shapin, Steven y Schaffer, Simon (1985): Leviathan and the Air Pump: Hobbes, Boyle and the Experimental Life, Princeton, Princeton UP.

Smolin, Lee (2007): The Trouble with Physics: The Rise of String Theory, the Fall of a Science, and What Comes Next, Londres, Penguin.

VAn FraAssen, Bas (1996): La imagen científica, Barcelona, Paidós.

WISE, Norton (2006): «Making Visible», Isis, 97, pp. 75-82.

YNDURÁIN, F. J. (2007): «Perspectivas para la física de partículas en el siglo XXI», Revista de Occidente, 319, pp. 23-45.

\section{RESUMEN}

El énfasis en la práctica científica, procedente originariamente de la sociología de la ciencia y, más recientemente, de la historia y la filosofía de la ciencia, está produciendo una concepción de la ciencia dramáticamente nueva. La práctica científica dibuja dentro de la órbita de la ciencia no sólo teorías y modelos, sino también hechos, instrumentos, aparatos, máquinas y seres humanos. El objetivo de este artículo es analizar la perspectiva experimentalista de pensar la física cuántica. Explico tres puntos de vista: el sociológico de Andrew Pickering, el epistemológico de Ian Hacking y el ontológico de Gustavo Bueno; quienes buscan moverse de la representación a la experimentación y la manipulación. Lo que distingue estas concepciones de la tradicional es que no comprenden la ciencia como una construcción en términos conceptuales y lingüísticos sino como una actividad social y material. 


\title{
PALABRAS CLAVE
}

Nuevo Experimentalismo, Sociología del Conocimiento Científico, idiomas representacional y performativo, epistemología materializada.

\begin{abstract}
The emphases on scientific practice, coming originally from the sociology of science and, more recently, from the history and philosophy of science, is producing a dramatically new view of science. Scientific practice draws into the orbit of science not only theories and models but also facts, instruments, apparatus, machines and human beings. The aim of this paper is to analyse the experimentalist trend of thinking about quantum physics. I explain three points of view: the sociological by Andrew Pickering, the epistemological by Ian Hacking, and the ontological by Gustavo Bueno. They aim to turn from representation to experimentation and manipulation. What distinguishes these views from the traditional one is that they do not see science as construction in conceptual and linguistic terms but rather as a social and material activity.
\end{abstract}

\section{KEYWORDS}

New Experimentalism, Sociology of Scientific Knowledge, representational and performative idioms, materialized epistemology. 